\title{
Calibration-Free Relaxation-Based Multi-Color Magnetic Particle Imaging
}

\author{
Yavuz Muslu ${ }^{\circledR}$, Mustafa Utkur, Omer Burak Demirel, and Emine Ulku Saritas ${ }^{(1)}$
}

\begin{abstract}
Magnetic particle imaging (MPI) is a novel imaging modality with important potential applications, such as angiography, stem cell tracking, and cancer imaging. Recently, there have been efforts to increase the functionality of MPI via multi-color imaging methods that can distinguish the responses of different nanoparticles, or nanoparticles in different environmental conditions. The proposed techniques typically rely on extensive calibrations that capture the differences in the harmonic responses of the nanoparticles. In this paper, we propose a method to directly estimate the relaxation time constant of the nanoparticles from the MPI signal, which is then used to generate a multi-color relaxation map. The technique is based on the underlying mirror symmetry of the adiabatic MPI signal when the same region is scanned back and forth. We validate the proposed method via simulations, and via experiments on our in-house magnetic particle spectrometer setup at $10.8 \mathrm{kHz}$ and our in-house MPI scanner at $9.7 \mathrm{kHz}$. Our results show that nanoparticles can be successfully distinguished with the proposed technique, without any calibration or prior knowledge about the nanoparticles.
\end{abstract}

Index Terms-Magnetic particle imaging, multi-color MPI, nanoparticle relaxation, direct estimation, mirror symmetry.

\section{INTRODUCTION}

$\mathbf{M}$ AGNETIC Particle Imaging (MPI) is a new and rapidly developing imaging modality, that shows great potential in terms of resolution, contrast, and sensitivity [1]-[5]. This imaging technique exploits the nonlinear magnetization response of superparamagnetic iron oxide (SPIO) nanoparticles, which provides MPI with high-contrast imaging capabilities, without any signal from the human tissue [1], [6], [7].

Manuscript received January 2, 2018; revised March 12, 2018 accepted March 16, 2018. Date of publication March 22, 2018; date of current version July 31, 2018. This work was supported in part by the Scientific and Technological Research Council of Turkey under Grant TUBITAK 114E167, in part by the European Commission through FP7 Marie Curie Career Integration under Grant PCIG13-GA-2013618834, in part by the TUBA-GEBIP 2015 Program of the Turkish Academy of Sciences, and in part by the BAGEP 2016 Award of the Science Academy. (Corresponding author: Yavuz Muslu.)

Y. Muslu, M. Utkur, and O. B. Demirel are with the Department of Electrical and Electronics Engineering, Bilkent University, 06800 Ankara, Turkey, and also with the National Magnetic Resonance Research Center (UMRAM), Bilkent University, 06800 Ankara, Turkey (e-mail: ymuslu@ee.bilkent.edu.tr)

E. U. Saritas is with the Department of Electrical and Electronics Engineering, Bilkent University, 06800 Ankara, Turkey, also with the National Magnetic Resonance Research Center (UMRAM) Bilkent University, 06800 Ankara, Turkey, and also with the Neuroscience Program, Sabuncu Brain Research Center, Bilkent University, 06800 Ankara, Turkey.

Color versions of one or more of the figures in this paper are available online at http://ieeexplore.ieee.org.

Digital Object Identifier 10.1109/TMI.2018.2818261
MPI uses a combination of three different magnetic fields to obtain an image: a static selection field with a strong gradient is used to create a field free point (FFP). Then, a sinusoidal drive field is applied to move the FFP and scan a field-ofview (FOV). However, human safety limits restrict the size of the FOV covered by the drive field [8]-[12]. Hence, lowfrequency focus fields are used to shift the FFP to regions that cannot be covered with the small FOV due to the drive field alone [13]-[15].

There are two main image reconstruction methods in MPI. System Function Reconstruction (SFR) requires the calibration of the nanoparticle response and the imaging system, which is achieved by a time-consuming calibration scan that records the frequency response of the signal induced by the SPIOs located at all voxel locations in the FOV [16]-[19]. The alternative image reconstruction technique, $\mathrm{x}$-space reconstruction, does not require any calibration as it reconstructs the image by gridding the acquired signal to the instantaneous position of the FFP [20], [21]. The resulting X-space reconstructed images show a version of the SPIO distribution blurred by the point spread function (PSF) of the imaging system. Both of these image reconstruction techniques, with their own advantages and disadvantages, map the spatial distribution of the SPIOs.

Rahmer et al. [22] has recently demonstrated that different SPIO types can be distinguished via a multi-color MPI technique, and numerous applications have already been shown to benefit from this novel approach. Recently, there has been significant progress in using multi-color MPI for catheter tracking during cardiovascular interventions [23]. In such applications, one SPIO type is injected into the blood stream for vessel visualization, while the catheter is coated with a different type of SPIO for tracking purposes. A further advancement of this technique involved simultaneous tracking and steering of the catheter tip via using the magnetic fields in MPI [24], [25]. More recently, the temperature mapping capability of multicolor MPI has also been demonstrated [26].

The binding state of the SPIOs has been spectroscopically shown to change the relaxation behavior, as well [27]. In this regard, relaxation mapping can be used to probe cell or protein binding of SPIOs. Moreover, there have been efforts on drug delivery via nanocarriers [28], where multi-color MPI can offer additional tracking possibilities. Likewise, multi-color MPI can also be used to identify the characteristics of an environment such as viscosity and temperature, which are shown to affect the nanoparticle relaxation behavior significantly [29]-[34]. These conditions can be important tools for probing the changes in tissue environments, e.g., in the 
case of hyperthermia treatments. All of the aforementioned applications create an immense room for further experimental work and research.

To date, multi-color MPI has been realized via both the SFR and the X-space approaches. In the SFR approach, SPIOs were differentiated based on the differences in their harmonic responses, which were obtained using an extensive calibration procedure performed separately for each SPIO type [22]. For the x-space approach, Hensley et al. [35] demonstrated that $\mathrm{x}$-space reconstruction is capable of multi-color MPI via differentiating the relaxation behaviors of different SPIO types, if multiple measurements at different drive field amplitudes are utilized. Recently, we have presented a method for estimating an effective relaxation time constant from the MPI signal, with preliminary results on a Magnetic Particle Spectrometer (MPS) setup [36]. However, whether this method could work for multi-color imaging scenarios was not shown experimentally.

In this work, we present a novel, calibration-free multi-color MPI technique for $\mathrm{x}$-space MPI. This technique can generate a relaxation time constant map of SPIOs from a single scan at a single drive field amplitude. Here, calibration-free refers to SPIO identification through their relaxation times, which are estimated directly from the MPI signal without any prior information about the SPIOs. The proposed technique takes advantage of the back and forth scanning of a FOV for the direct estimation of the relaxation times. Here, we demonstrate with simulation results, MPS experiments, and imaging experiments that the proposed method successfully distinguishes multiple SPIO types, without requiring any calibrations.

\section{THEORY}

\section{A. MPI Signal}

In $\mathrm{x}$-space MPI, the ideal signal (also known as the adiabatic signal) is defined via the Langevin response of the nanoparticles to an applied drive field [20]:

$$
s_{\text {adiab }}(t)=\left.B_{1} m \rho(x) * \dot{\mathcal{L}}[k G x]\right|_{\mathrm{x}=\mathrm{x}_{\mathrm{s}}(t)} k G \dot{x}_{\mathrm{s}}(t)
$$

Here, $B_{1}[\mathrm{~T} / \mathrm{A}]$ is the receive coil sensitivity, $m\left[\mathrm{Am}^{2}\right]$ is the magnetic moment of nanoparticles, $\rho(x)$ [particles $/ \mathrm{m}^{3}$ ] is the nanoparticle density, $k[\mathrm{~m} / \mathrm{A}]$ is a nanoparticle property defined as the inverse of the field required for saturation, $x_{\mathrm{s}}(t)[\mathrm{m}]$ is the instantaneous position of the FFP, $\dot{x}_{\mathrm{s}}(t)[\mathrm{m} / \mathrm{s}]$ is the FFP velocity, and $G\left[\mathrm{~T} / \mathrm{m} / \mu_{0}\right]$ is the gradient strength of the selection field [20], [21]. In practice, the MPI signal lags and gets wider due to magnetic nanoparticle relaxation effects, i.e., the delay of SPIOs in aligning with oscillating magnetic fields (see Fig.1b). Magnetic nanoparticle relaxation is modelled as a first-order Debye process, which corresponds to the temporal convolution of the ideal MPI signal with an exponential kernel in x-space MPI [37]-[39]:

$$
\begin{aligned}
& s(t)=s_{\text {adiab }}(t) * r(t) \\
& r(t)=\frac{1}{\tau} e^{-\frac{t}{\tau}} u(t)
\end{aligned}
$$

Here, $r(t)$ denotes the relaxation kernel, $u(t)$ is the Heaviside step function stepping at $t=0$, and $\tau$ is the relaxation time constant. The resulting MPI signal is called the non-adiabatic
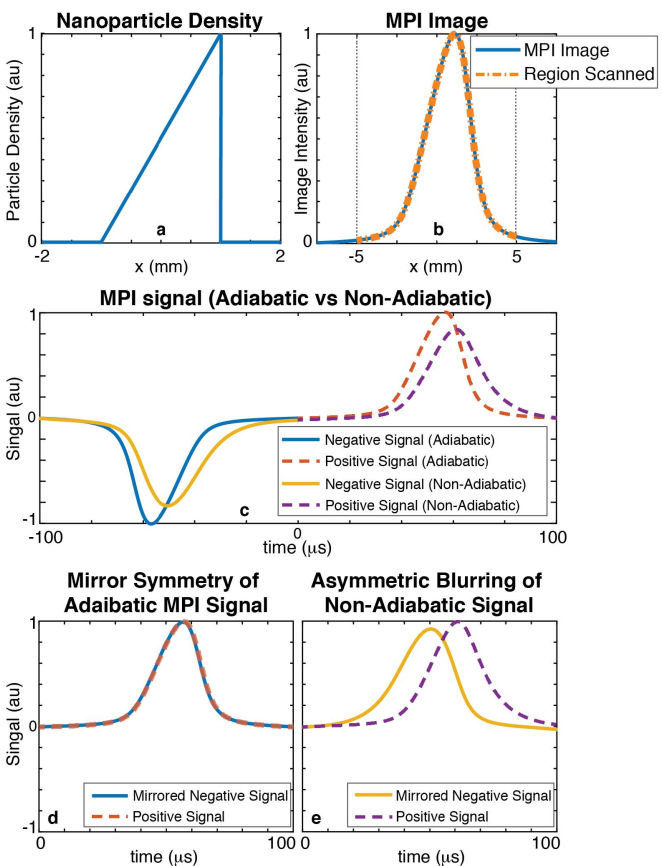

Fig. 1. The mirror symmetry of the adiabatic MPI signal and how the relaxation effects break that mirror symmetry. (a) A ramp-shaped nanoparticle density. (b) The corresponding ideal MPI image obtained by the convolution of the nanoparticle density and the point spread function. (c) Adiabatic vs. non-adiabatic MPI signals for the region scanned. Here, one full periods of the signals are shown (i.e., the signals from both the negative and positive cycles). (d) In the adiabatic case, due to the repetitive nature of the applied sinusoidal drive field, the positive and mirrored negative signals match perfectly. We refer to this phenomenon as "mirror symmetry". (e) The relaxation effect causes an asymmetric blurring of the negative and positive signals, which in turn breaks the mirror symmetry.

signal. Via extensive experimental studies, this simple yet powerful model has been shown to accurately match the relaxation effect for a wide range of drive field frequencies and amplitudes [37], [40]. It is important to note that, $\tau$ in Eq. 3 does not correspond to Neel or Brownian relaxation time constants. Instead, it is a lumped parameter that explains the lagging and widening of the MPI signal due to the delayed response of the nanoparticles.

\section{B. Theory of Direct $\tau$ Estimation}

In MPI, a sinusoidal drive field is applied to excite the SPIOs, while additional focus fields are applied to control the global position of the FFP. For a constant focus field amplitude, the resulting FFP trajectory scans a partial fieldof-view (pFOV) back and forth around a central position. For simplicity, the forward motion of the FFP is called the positive scan, while the backward motion is called the negative scan. In Fig. 1a-b, a ramp-shaped SPIO density and the corresponding MPI image are given. For the back and forth trajectory, one would expect the signals acquired during these two scans to be mirror symmetric or point symmetric (i.e., symmetric with respect to the central time point of one period), as displayed in Fig. 1c-d. However, the nanoparticle relaxation causes an asymmetric blurring that breaks the mirror symmetry, as shown in Fig.1e. 
We have previously introduced a technique to directly estimate the relaxation time constant from the MPI signal, using the underlying mirror symmetry of the adiabatic signal [33], [36], [41]. In this technique, we formulated the effects of relaxation in Fourier domain. Accordingly, we define $s_{\text {pos }}(t)$ as the signal acquired during the positive scan, and $s_{\text {neg }}(t)$ as the signal acquired during the negative scan, both centered with respect to time. For the adiabatic MPI signal, $s_{\text {pos,adiab }}(t)$ and $s_{\text {neg,adiab }}(t)$ are mirror symmetric, i.e.,

$$
s_{\text {pos,adiab }}(t)=-s_{\text {neg,adiab }}(-t)=s_{\text {half }}(t)
$$

where, $s_{\text {half }}(t)$ denotes half a period of the adiabatic MPI signal. Using the relaxation formulation in Eq. 2, the nonadiabatic positive and negative signals can be expressed as:

$$
\begin{aligned}
& s_{\text {pos }}(t)=s_{\text {pos,adiab }}(t) * r(t)=s_{\text {half }}(t) * r(t) \\
& s_{\text {neg }}(t)=s_{\text {neg,adiab }}(t) * r(t)=-s_{\text {half }}(-t) * r(t)
\end{aligned}
$$

Here, $s_{\text {half }}(t)$ and $r(t)$ are the unknowns of the equations while $s_{\text {pos }}(t)$ and $s_{\text {neg }}(t)$ are measured waveforms. In Fourier domain, these equations can be expressed as:

$$
\begin{aligned}
\mathcal{F}\{r(t)\} & =R(f)=\frac{1}{1+i 2 \pi f \tau} \\
\mathcal{F}\left\{s_{\text {pos }}(t)\right\} & =S_{\text {pos }}(f)=S_{\text {half }}(f) \cdot R(f) \\
\mathcal{F}\left\{s_{\text {neg }}(t)\right\} & =S_{\text {neg }}(f)=-S_{\text {half }}^{*}(f) \cdot R(f)
\end{aligned}
$$

where $\mathcal{F}$ is the Fourier transform operator, and the time reversal and conjugate symmetry properties of Fourier Transform are used to express $S_{\text {neg }}(f)$. Using Eqs. 7-9, $\tau$ can be calculated directly as follows:

$$
\tau(f)=\frac{S_{\mathrm{pos}}^{*}(f)+S_{\text {neg }}(f)}{i 2 \pi f\left(S_{\mathrm{pos}}^{*}(f)-S_{\text {neg }}(f)\right)}
$$

Ideally, performing this calculation at a single frequency in Fourier domain suffices. However, the accuracy of the estimation is different at each frequency component due to relative strengths of signal vs. noise. To increase the robustness of the estimation against noise, a weighted average of $\tau(f)$ is calculated with respect to the magnitude spectrum:

$$
\tau=\frac{\int_{0}^{f_{\max }}\left|S_{\mathrm{pos}}(f)\right| \tau(f) d f}{\int_{0}^{f_{\max }}\left|S_{\mathrm{pos}}(f)\right| d f}
$$

Here, $f_{\max }$ is an upper threshold for the range of frequencies used, such that $0<f_{\max } \ll \frac{F_{\mathrm{s}}}{2}$, where $F_{\mathrm{s}}$ is the sampling frequency. Typically, including frequencies up to $6^{\text {th }}$ or $7^{\text {th }}$ harmonic of the fundamental frequency suffices, as the signal falls off rapidly with increasing frequency.

\section{Materials and Methods}

\section{A. Verification of Direct $\tau$ Estimation}

The direct relaxation time constant estimation method is tested in MATLAB (Mathworks, Natick, MA). Figure 2 demonstrates the proposed relaxation time constant estimation method on a simulated MPI signal. Here, the same rampshaped nanoparticle density in Fig. 1a is assumed and a central pFOV is scanned to obtain the MPI signal. Simulation parameters were as follows: $15 \mathrm{mT}$-peak drive field at $5 \mathrm{kHz}$,



Fig. 2. The proposed method: direct relaxation time constant estimation. (a) The mirror symmetry between the positive and negative signals is broken due to relaxation. The proposed method estimated $\hat{\tau}=6.96 \mu \mathrm{s}$, where $\tau=7 \mu \mathrm{s}$ in theory. (b) MPI signal is then deconvolved with the estimated relaxation kernel using $\hat{\tau}=6.96 \mu \mathrm{s}$, which recovers the underlying mirror symmetry. Here, $(a, b)$ demonstrate the case where the fundamental frequency is kept intact in simulations, and (c, d) demonstrate the case after direct feedthrough filtering that removes the fundamental frequency. In the latter case, the estimation resulted in $\hat{\tau}=6.99 \mu s$.

$3 \mathrm{~T} / \mathrm{m} / \mu_{0}$ gradient, $2 \mathrm{MS} / \mathrm{s}$ sampling frequency, $21 \mathrm{~nm}$ nanoparticle diameter, and $7 \mu$ s relaxation time constant. First, the MPI signal is simulated using a very small discretization time step of $1 \mathrm{~ns}$. The relaxation effect is then incorporated via the exponential model in Eq. 2. Next, this MPI signal is resampled with the sampling frequency of our data acquisition card $(2 \mathrm{MS} / \mathrm{s})$ to ensure that the results are realistic.

For the MPI signal with the fundamental frequency, the estimated time constant yielded $\hat{\tau}=6.96 \mu \mathrm{s}$. The signal was then deconvolved using the estimated relaxation kernel, recovering the underlying mirror symmetry (see Fig. 2a-b). The same procedure was repeated on the MPI signal after direct feedthrough filtering [6], [42], to ensure that the filtering of the fundamental harmonic (a necessary step in MPI) does not hinder the performance of the proposed technique, where the estimated time constant yielded $\hat{\tau}=6.99 \mu$ s (see Fig. 2c-d). In the absence of noise in both cases, the proposed method estimated $\tau$ with less than $0.6 \%$ error. This error increases for larger discretization time steps (e.g., $2 \%$ error for $20 \mathrm{~ns}$ ) and steadily converges to zero for smaller time steps. Hence, we conclude that this error is primarily caused by the discretization in simulating the relaxation effect via convolution.

\section{B. Experimental Relaxation Time Constant Estimation}

Finding the correct time intervals for $s_{\text {pos }}(t)$ and $s_{\text {neg }}(t)$ is crucial for the proposed relaxation time estimation method. In simulations, this procedure is trivial since the timing of the signal vs. the FFP trajectory (i.e., the time point, $t_{\text {edge }}$, when the positive scan ends and the negative scan starts) is known. In practice, however, system delays introduce a time 
shift between the signal vs. the FFP trajectory. In such cases, incorrect $t_{\text {edge }}$ values introduce extra phase terms in Eq. 10 (see Supplementary Materials ${ }^{1}$ ), causing incorrect estimation of $\tau$. One method for measuring $t_{\text {edge }}$ is performing a calibration scan with an SPIO that exhibits negligible relaxation (e.g., fluidMAG nanoparticles by Chemicell, as suggested in [37]). Here, we propose a procedure that estimates the correct $\tau$ and $t_{\text {edge }}$ simultaneously, eliminating the need for a fine-tuned calibration scan.

We have argued previously that deconvolution of the MPI signal with the correct relaxation kernel should restore mirror symmetry. Here, we exploit this phenomenon to simultaneously find the correct $\tau$ and $t_{\text {edge, }}$ as outlined in Fig. 3. First, we choose a $t_{\text {edge }}$ value, directly estimate $\tau$ using Eq. 11, and deconvolve the MPI signal with the estimated relaxation kernel. We then repeat this step for a range of $t_{\text {edge }}$ values limited to $[0, T / 2)$ region of the MPI signal, where $T$ is the drive field period. The correct pair, $\left(\hat{\tau}, \hat{t}_{\text {edge }}\right)$, should restore the mirror symmetry, minimizing the mean squared error (MSE) between positive and mirrored negative signals after deconvolution, i.e.,

$$
\left(\hat{\tau}, \hat{t}_{\text {edge }}\right)=\underset{\left(\tau, t_{\text {edge }}\right)}{\operatorname{argmin}} \int_{0}^{T / 2}\left(\hat{s}_{\text {pos }}(t)-\left(-\hat{s}_{\text {neg }}(-t)\right)^{2} d t\right.
$$

where $\hat{s}_{\text {pos }}(t)$ and $\hat{s}_{\text {neg }}(t)$ depend on $\tau$ and $t_{\text {edge, }}$, and denote the signals after deconvolution with the estimated relaxation kernel. A detailed analysis showing that Eq. 12 has a unique minimum is given in Fig. S1 in Supplementary Materials. Here, instead of computing MSE over the entire half period, more weights can be assigned to central time points that typically have higher signal-to-noise ratio (SNR) [33]. In addition, the search for $t_{\text {edge }}$ could also be performed in $(-T / 2,0]$, which would yield an identical $\hat{\tau}$ value.

\section{Proposed Algorithm: Calibration-Free Relaxation Mapping}

For the proposed multi-color MPI technique, we directly estimate a relaxation time constant for each $\mathrm{pFOV}$, and map the estimated time constant to the central position of the pFOV. While this mapping sounds simple at first, extensive simulations revealed two special cases where additional steps are needed for accurate mapping of time constants:

1) Flat Nanoparticle Distribution: If the nanoparticle distribution is flat in a pFOV, most of the signal is contained in the lost $1^{\text {st }}$ harmonic. Hence, direct feedthrough filtering removes almost all signal, making flat regions appear like regions devoid of nanoparticles. In the absence of signal, the proposed estimation method produces noiselike results.

2) Inhomogeneous Mixtures of Different Nanoparticle: In the regions where two different nanoparticle types mix homogeneously, the estimation yields a weighted average of two relaxation time constants (see Appendix). However, in the case of an inhomogeneous mixture (e.g.,

\footnotetext{
${ }^{1}$ The detailed derivation of the extra phase terms and their effects on the estimations can be found in the Supplementary Materials available in the supplementary files/multimedia tab.
}

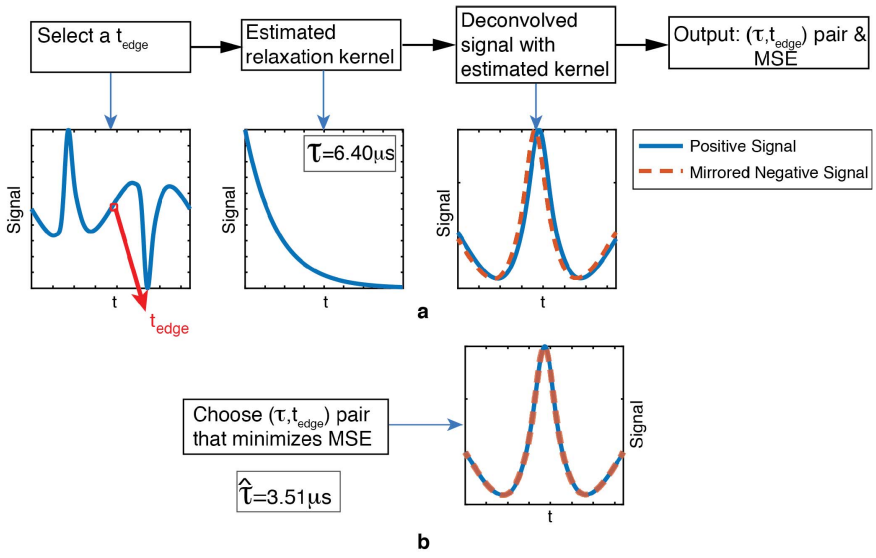

Fig. 3. Experimental relaxation time constant estimation algorithm. (a) A time point $t_{\text {edge }}$ is chosen to mark the time when positive scan finishes and negative scan starts. Then, $\tau$ is estimated for different $t_{\text {edge }}$ values chosen from [0, T/2) interval. The MSE between the deconvolved positive and mirrored negative signals are computed for each case. (b) The MSE values are compared, and the $\left(\tau, t_{\text {edge }}\right)$ pair that minimizes the MSE is chosen as the solution. For this example, the result is $\hat{\tau}=3.51 \mu \mathrm{s}$. Here, the black arrows show the flow of the algorithm, while blue arrows show a visual demonstration of each step.

a transition region from one nanoparticle type to the other), the adiabatic signal from each nanoparticle not only has a different shape, but is also convolved with a different relaxation kernel. Therefore, the set of equations derived in Eqs. 8-9 become underdetermined, producing noise-like estimations.

Here, we first detect and isolate these special cases, reconstruct the rest of the relaxation map, and finally restore the missing portions of the map. The following steps summarize the proposed multi-color MPI algorithm, outlined in Fig. 4 for an example case of Inhomogeneous Mixtures:

Phase 0 - Estimation of $\tau$ for Each pFOV: Using Eq. 12, the algorithm directly estimates the corresponding $\left(\hat{\tau}, \hat{t}_{\text {edge }}\right)$ pair for each pFOV. An $\mathrm{x}$-space MPI image is reconstructed (Fig. 4b), and the signal root mean squared (RMS) values for $\mathrm{pFOVs}$ are calculated using all samples from one period of the corresponding signals (Fig. 4c).

Phase 1 - Histogram Correction: Using the histogram of estimated $\tau$ values from all pFOVs, a mild thresholding is applied to remove erroneous estimations. The histogram correction consists of 2 steps (see Fig. 4d-e):

- Upper limit on estimations: Extensive experimental work showed that $\tau$ values are smaller than $10 \%$ of the drive field period for the exponential model [33], [40]. Here, as a safe upper limit, we ignore estimations that are larger than one-fourth of the period.

- Signal RMS threshold: Low signal leads to inaccurate $\tau$ estimations. Here, estimations from low RMS regions (e.g., less than $10 \%$ of the maximum RMS value) are initially ignored.

Phase 2 - Cluster Detection: At the end of Phase 1, erroneous estimations have been mostly removed. Accordingly, the remaining estimations must have the relaxation times of different SPIO types. Here, a k-means clustering algorithm is 


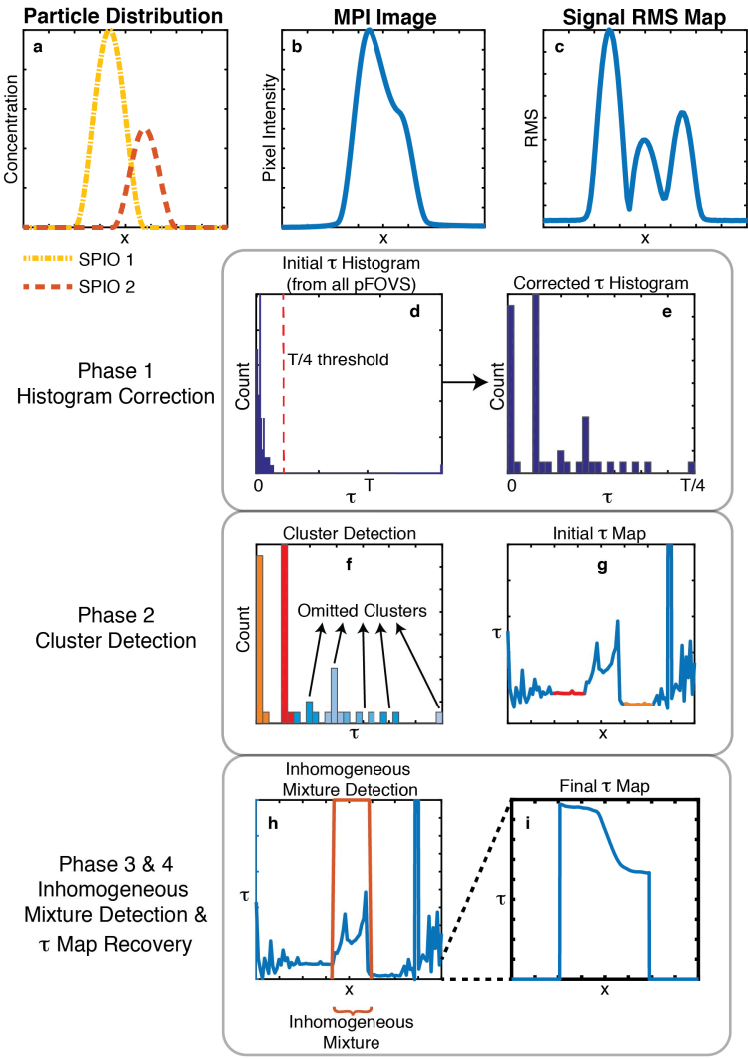

Fig. 4. The proposed relaxation mapping algorithm. (a) The simulated particle distributions for two different SPIO types, which overlap in a small region in 1D space. (b) The $\mathrm{x}$-space reconstructed MPI image. (c) Signal RMS values for each pFOV. (d) The initial relaxation time constant estimations are plotted as a histogram, and (e) the histogram is corrected by eliminating low SNR regions and overestimations (i.e., an upper threshold set at T/4). (f) A k-means clustering detects two main clusters (shown in orange and red). (g) These regions are highlighted in the initial $\tau$ map. (h) The inhomogeneous nanoparticle mixture region is detected, and (i) the final $\tau$ map is achieved via modeling the transition using a smooth sigmoid curve and setting low pixel intensity regions to zero. Note the change in $y$-axis scaling from (h) to (i).

run on the remaining estimations [43]. Since the number of nanoparticle types is unknown, $k$ parameter is started from a large value. The following procedure is then applied iteratively to reduce $k$, with the goal of representing the probable SPIO types (see Fig. 4f-g):

- Merger of clusters: Cluster centers that are closer than a $\tau_{\text {res }}$ value are merged. Note that $\tau_{\text {res }}$ must be sufficiently small to ensure the separation of different clusters.In our simulations, $\tau_{\text {res }}$ is selected as $T / 100$.

- Omission of small clusters: Clusters that contain less than $p \%$ of the overall histogram are considered irrelevant and ignored. In our simulations, $p$ is taken as 5 .

- Convergence: If the cluster properties (number of clusters, cluster centers, etc.) remain the same for two consecutive iterations, convergence is achieved.

Note that the purpose of this step is to find the range of probable $\tau$ values for each SPIO type and eliminate outlier estimations. Individual $\tau$ variations within each SPIO type are still preserved.
Phase 3 - Special Case Detection: After Phase 2, clusters have been detected. Outlier $\tau$ values that are not in the vicinity of the clusters could indicate either one of the two special cases explained above. These cases have high pixel intensity in the MPI image despite having low signal RMS. Here, we search for the nearest spatial locations that have $\tau$ values belonging to a cluster, on either side of the problem pFOV. If incorrect estimations are caused by inhomogeneous mixtures, we expect different clusters on either sides of the problem pFOVs (see Fig. 4h). On the other hand, flat nanoparticle distributions have a single cluster type around the problem pFOVs.

Phase 4-Recovery of $\tau$ Map: At the end of Phase 3, the regions of incorrect estimations are determined. These regions are recovered as follows (see Fig. 4i):

- For Flat Nanoparticle Distributions, the missing regions are recovered as a constant $\tau$ value.

- For Inhomogeneous Mixtures, the average signal RMS of the clusters are used to estimate the relative concentrations of the corresponding nanoparticle types. Next, a smooth transition region from one relaxation time to the other is modeled using a sigmoid function, with steepness determined via the relative concentrations.

Finally, estimations from low MPI pixel intensity regions are set to zero to remove the background in the $\tau$ map.

\section{1D and Multidimensional Simulations}

All simulations were carried out using a custom MPI toolbox developed in MATLAB (Mathworks, Natick, MA). To obtain realistic results, the fundamental harmonic was filtered out [6], the relaxation time constant values were selected according to previous studies [33], [40], and smoothlyvarying Hamming-window-shaped SPIO distributions were used to simulate real-life conditions. The MPI images were reconstructed using the $\mathrm{x}$-space reconstruction technique [6], [20], [21].

For $1 \mathrm{D}$ simulations, a selection field with $3 \mathrm{~T} / \mathrm{m} / \mu_{0}$ gradient was utilized, with $10 \mathrm{mT}$-peak drive field at $10 \mathrm{kHz}$ and $80 \%$ overlap between consecutive pFOVs. The nanoparticle diameter was selected as $21 \mathrm{~nm}$ (a conservative value given the recent developments in tailored SPIOs with larger diameter and narrower PSF [44]) to test the proposed methods under less ideal conditions. The relaxation times ranged between $1-5 \mu \mathrm{s}$, as recently reported in experimental studies performed around $10 \mathrm{kHz}$ [33], [40]. To match experimental conditions, the simulated MPI signal was sampled at $2 \mathrm{MS} / \mathrm{s}$ sampling frequency, and additive white Gaussian noise corresponding to a peak SNR of 5 was added to the MPI signal.

For multidimensional simulations, a selection field with $(-7,3.5,3.5) \mathrm{T} / \mathrm{m} / \mu_{0}$ gradient in $(\mathrm{x}, \mathrm{y}, \mathrm{z})$ directions was utilized, based on the work in [6]. A 15 mT-peak drive field at $10 \mathrm{kHz}$ and a 90\% overlap along the z-direction was utilized, with all other parameters kept the same as in 1D simulations. Here, the drive field was 1D in the z-direction only, which enabled a line-by-line scanning of a $2 \mathrm{D}$ FOV in the $\mathrm{x}-\mathrm{z}$ plane. The corresponding 2D relaxation map was obtained by stacking the 1D relaxation maps for the scanned lines. 


\section{E. MPS Experiments}

The initial experiments of the proposed relaxation time estimation method were conducted on our in-house MPS (also known as MPI Relaxometer) setup. The drive coil of the relaxometer produces $0.97 \mathrm{mT} / \mathrm{A}$ magnetic field with $95 \%$ homogeneity in a $7-\mathrm{cm}$ long region. The receive coil of the relaxometer was designed as a three-section gradiometer pickup coil [33], to minimize the direct feedthrough signal. A more detailed explanation of the setup can be found in [33].

Experiments were conducted at $10.8 \mathrm{kHz}$ and $15 \mathrm{mT}$-peak drive field. The nanoparticle signal was first amplified with a low-noise voltage preamplifier (Stanford Research Systems SR560), then digitized at $2 \mathrm{MS} / \mathrm{s}$ via a data acquisition card (National Instruments, NI USB-6363). 16 consecutive signal acquisitions were performed, each with $30 \mathrm{~ms}$ drive field pulse duration. The received signals were averaged, providing a 4-fold SNR improvement. A background measurement was subtracted from the averaged signal to minimize the effects of potential background interferences in the higher harmonics of the fundamental frequency. A frequency domain filter was applied by selecting the higher harmonics of the drive field and setting the rest of the frequency components to zero in Fourier domain. Next, a high-order zero-phase digital low pass filter (LPF) was applied in time domain to avoid ringing artifacts. The cut-off frequency of the LPF was determined by setting an SNR threshold of 2 in frequency domain, and avoiding the $250 \mathrm{kHz}$ self-resonance frequency of the receive coil, which corresponded to a cut-off frequency of $180 \mathrm{kHz}$. For the deconvolution step of the proposed method, we used Wiener deconvolution and chose a relatively large noise to signal power ratio of 100 to prevent noise amplification.

For MPS experiments, VivoTrax ferucarbotran nanoparticles (Magnetic Insight Inc., USA) with the same chemical composition as Resovist, and Nanomag-MIP nanoparticles (Micromod GmbH, Germany) were prepared in $25 \mu \mathrm{L}$ volumes. The original concentrations of Nanomag-MIP and VivoTrax were $5.5 \mathrm{mg} \mathrm{Fe} / \mathrm{mL}$ and $5 \mathrm{mg} \mathrm{Fe} / \mathrm{mL}$, respectively. The Nanomag-MIP sample was diluted 3.5 times to equalize its peak signal level with that of the VivoTrax sample. In addition, a $50 \%-50 \%$ homogeneous mixture of the two samples was prepared in $25 \mu \mathrm{L}$ volume.

\section{F. Imaging Experiments}

Our in-house MPI scanner (see Fig. 5) has two disc-shaped permanent magnets with $2-\mathrm{cm}$ thickness and 7-cm diameter, placed at $8-\mathrm{cm}$ separation in the $\mathrm{x}$-direction. The resulting configuration creates $(-4.8,2.4,2.4) \mathrm{T} / \mathrm{m} / \mu_{0}$ gradient in $(\mathrm{x}, \mathrm{y}, \mathrm{z})$ directions, which yields approximately $4-\mathrm{mm}$ resolution in the z-direction (i.e., down the imaging bore) for NanomagMIP nanoparticles. The drive coil has 3 layers of Litz wire with 80 turns, resulting in $1.5 \mathrm{mT} / \mathrm{A}$ magnetic field with $95 \%$ homogeneity in a $4.5 \mathrm{~cm}$-long region down its bore. The drive field and selection field specifications were validated using a Hall Effect Gaussmeter (LakeShore 475 DSP Gaussmeter). The receive coil was designed as a three-section gradiometer, with a single layer of Litz wire with 34 turns in the main section and 17.5 turns in the side sections. The self-resonance of the receive coil was measured at around $280 \mathrm{kHz}$. This

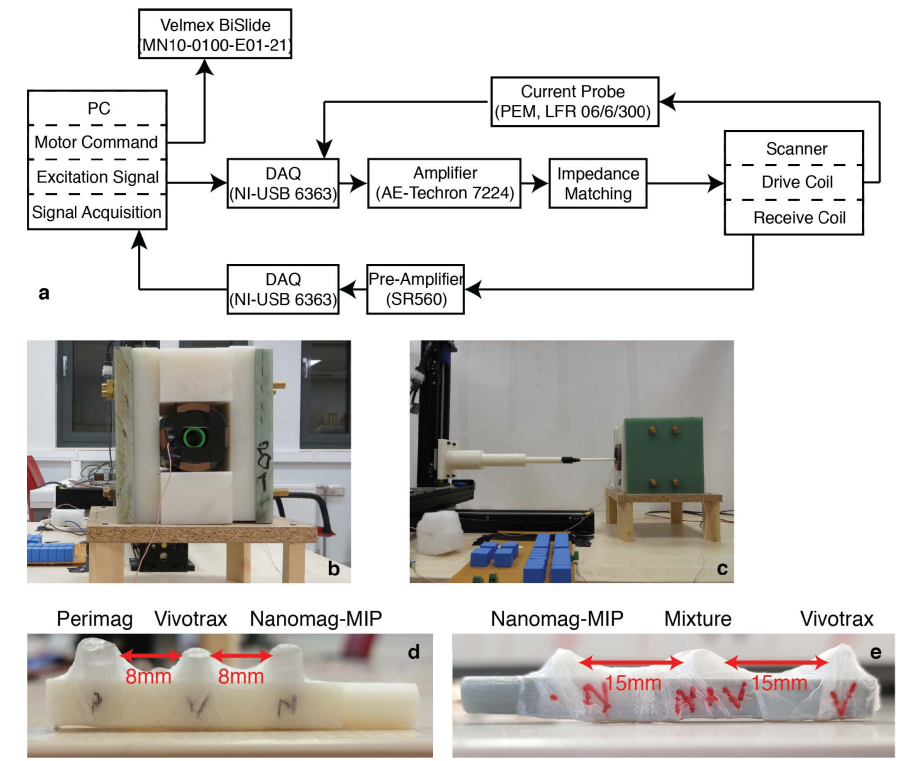

Fig. 5. An overview of the MPI scanner and the experimental setup. (a) The entire imaging system is controlled through MATLAB, via a custom MPI imaging toolbox. First, the robot position is adjusted to place the phantom at the desired location. Second, a drive field at $9.7 \mathrm{kHz}$ is applied through the transmit chain, and the nanoparticle signal is simultaneously acquired through the receive chain. (b-c) The front and side views of our in-house MPI scanner. The phantoms imaged in (d) the $1 \mathrm{D}$ experiment and $(\mathrm{e})$ the $2 \mathrm{D}$ experiment.

configuration allowed $1 \times 1 \times 10 \mathrm{~cm}^{3}$ FOV. The drive and receive coils were placed inside a cylindrical copper shield with 1-cm thickness (to allow potential imaging applications at drive field frequencies as low as $1 \mathrm{kHz}$ ).

Figure 5a-c display the workflow of the complete MPI scanner setup, together with its front and side views. The overall imaging system was controlled via a custom toolbox implemented in MATLAB (Mathworks, Natick, MA). The drive coil was impedance matched to a power amplifier (AE Techron 7224), using a capacitive network at $9.7 \mathrm{kHz}$. The imaging phantoms were mechanically moved inside the scanner in 3D via Motor-Driven Velmex BiSlide (Model: MN100100-E01-21).

The drive field was at $15 \mathrm{mT}$-peak and $9.7 \mathrm{kHz}$, which resulted in a $12.5 \mathrm{~mm}$ pFOV length in the z-direction. The amplitude of the drive field was automatically calibrated immediately before each experiment and controlled throughout the experiment via a Rogowski current probe (LFR 06/6/300, PEM Ltd.). Partial FOVs were acquired with $85 \%$ overlaps in the $\mathrm{z}$-direction. For the 1D imaging experiment, an $8-\mathrm{cm}$ FOV along the $\mathrm{z}$-direction was covered with $16.8 \mathrm{sec}$ active scan time. For the 2D imaging experiment, a Cartesian trajectory was used to cover a $0.8 \times 8 \mathrm{~cm}^{2}$ FOV in the $\mathrm{X}-\mathrm{z}$ plane using 9 lines along the $\mathrm{z}$-direction, with $151 \mathrm{sec}$ active scan time. The temperature inside the scanner bore was controlled throughout the experiment to prevent heating of the nanoparticles.

To boost the SNR, 16 consecutive signal acquisitions were performed, each with $30 \mathrm{~ms}$ drive field pulse duration. The received signals were averaged, providing a 4-fold SNR improvement. The background measurements acquired before/after each line were subtracted from the nanoparti- 
cle signal to minimize potential background interferences. All remaining signal acquisition, processing, and deconvolution steps were the same as in the MPS experiments. The MPI images were reconstructed using $\mathrm{x}$-space reconstruction [6], [20], [21], followed by the proposed multi-color MPI technique.

\section{G. Imaging Phantom Preparation}

Imaging phantoms with different types of SPIOs were prepared, using VivoTrax (Magnetic Insight Inc., USA), and Perimag and Nanomag-MIP nanoparticles (Micromod GmbH, Germany). These nanoparticles had original concentration levels of $5.5 \mathrm{mg} \mathrm{Fe} / \mathrm{mL}, 17 \mathrm{mg} \mathrm{Fe} / \mathrm{mL}$, and $5 \mathrm{mg} \mathrm{Fe} / \mathrm{mL}$, respectively. Perimag and Nanomag-MIP SPIOs were diluted 10.2 and 3.5 times, respectively, to approximately equalize their MPS signal levels to that of VivoTrax. This equalization was performed solely for visual reasons: to have comparable pixel intensities from these nanoparticles in the $\mathrm{x}$-space MPI image. Capillary tubes with $2 \mathrm{~mm}$ inner diameter were filled with $10 \mu \mathrm{L}$ volume of SPIOs each. These tubes were then placed in custom-designed 3D-printed phantom holders in two different configurations, as shown in Fig. 5d-e. One of the tubes in the 2D experiment contained a 50\%-50\% homogeneous mixture of Nanomag-MIP and VivoTrax SPIOs.

\section{Results}

\section{A. 1D and Multidimensional Simulation Results}

Figure 6 displays the results of the proposed mapping algorithm for the 1D case, where each row corresponds to a different scenario: two, three, and four different types of SPIOs, respectively. The left column shows the spatial distributions of SPIOs with relaxation times ranging between 1-5 $\mu \mathrm{s}$. The MPI images, reconstructed via the $\mathrm{x}$-space reconstruction, are displayed in the middle column. Each simulation includes a challenging case of inhomogeneous mixtures of different nanoparticle types. The right column shows the reconstructed relaxation maps (blue solid lines) and the ideal relaxation maps (orange dashed lines). The ideal maps were calculated on a pixel-by-pixel basis by averaging $\tau$ values of SPIO constituents weighted by their relative pixel intensities. While utilizing only two different SPIO types is more likely in medical applications (e.g., coated catheter tip vs. blood pool in the case of cardiovascular interventions [25]), these results aim to show the full potential of the proposed method.

As seen in each row in Fig. 6, the proposed mapping algorithm reconstructed the relaxation maps accurately, despite a relatively low SNR level of 5 (see Fig. S2 and S3 in Supplementary Materials for a detailed noise robustness analysis). The inhomogeneous mixture regions were detected successfully and the proposed sigmoid-transition model recovered those regions accurately. Furthermore, the proposed algorithm also eliminated background areas via a thresholding and edge detection step. The slight deviations from the ideal relaxation maps were due to noise, as well as the crosstalk of signals from different SPIOs due to proximity. For the results in Fig. 6c, the mean estimation error is well below $3 \%$, whereas the mean error reaches $7 \%$ for the results in Fig. 6i where the SPIO distributions are closer to each other. Here, the 3.5- $\mu$ s SPIO
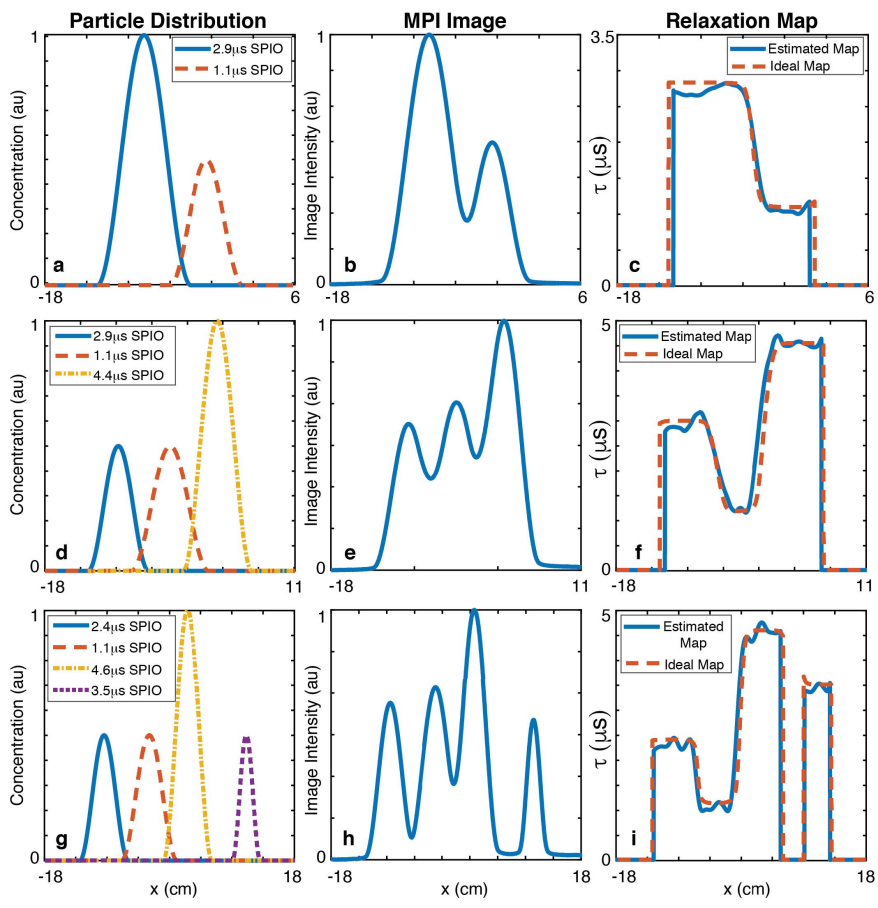

Fig. 6. Results of 1D simulations for three different cases and SNR level of 5 , without signal averaging. Left column $(a, d, g)$ demonstrates the spatial distributions for different SPIOs with relaxation time constants ranging between 1-5 $\mu \mathrm{s}$. The middle column $(\mathrm{b}, \mathrm{e}, \mathrm{h})$ demonstrates the corresponding $x$-space MPI images. The right column (c, f, i) demonstrates the relaxation maps reconstructed with the proposed algorithm. The mean estimation error is well below $3 \%$ for (c), and is around $7 \%$ for (i) due to reduced distances between the different SPIO distributions.

was spatially disconnected from the other SPIOs, which is successfully depicted in the reconstructed relaxation map.

For the multidimensional case, the proposed technique is extended in a line-by-line basis, where each line in the z-direction is reconstructed individually, then combined to form the multidimensional multi-color MPI image. In Fig. 7a, each SPIO distribution (labeled from 1 to 9) represents a homogeneous mixture of two different SPIOs with $2.9 \mu$ s and $1.1 \mu \mathrm{s}$ relaxation time constants at different mixture ratios. Accordingly, the distribution labeled as 1 has $100 \%$ of $2.9-\mu \mathrm{s}$ SPIOs, whereas the distribution labeled as 9 has $100 \%$ of 1.1 $\mu$ s SPIOs. Figure $7 \mathrm{~b}$ shows the resulting $\mathrm{x}$-space MPI image, and Fig. 7c shows the relaxation map color overlay, which was obtained by multiplying the $\mathrm{x}$-space MPI image and the relaxation map. In the MPI image, the regions corresponding to larger $\tau$ values resulted in slightly lower pixel intensities due to relaxation-induced signal losses. However, this difference cannot be used to distinguish the SPIOs, as lower iron concentration could also result in reduced pixel intensity. As seen in Fig. 7c, the proposed multi-color MPI method is capable of distinguishing a variety of SPIO types ( 9 in this case), each with a different time constant. Furthermore, the estimated time constants reflect the relative concentrations of the constituent SPIO types with a high level of linearity $\left(R^{2}=0.9921\right)$, as shown in Fig. 7d.

\section{B. MPS Experiment Results}

Figure 8 presents the experimental validation for the proposed relaxation time constant estimation technique and the 

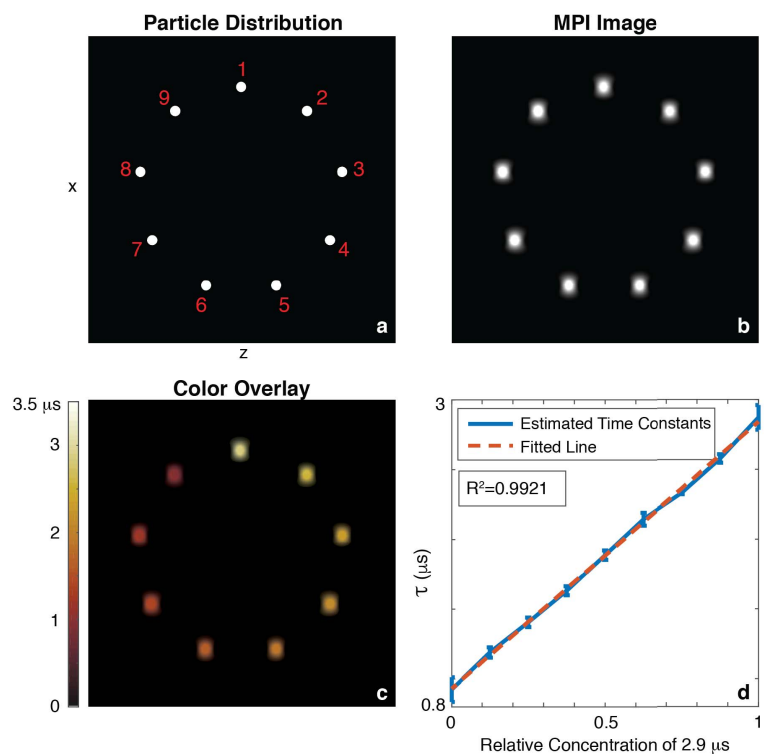

Fig. 7. Results of 2D simulations, for SNR level of 5. (a) A distribution with homogeneous mixtures of $2.9 \mu \mathrm{s}$ and $1.1 \mu \mathrm{s}$ SPIOs in different concentrations. Each mixture is labeled with a number from 1 to 9 , with concentrations of $2.9 \mu \mathrm{s}$ SPIO in each mixture given as $(100,87.5,75$, $62.5,50,37.5,25,12.5,0) \%$, respectively. (b) The x-space MPI image, and (c) the color overlay of the multi-color relaxation map and the MPI image. (d) The estimated time constants vs. relative concentrations of the two SPIOs. Here, the error bars denote the standard deviations for each $\mathrm{ROI}$, and the red dashed curve is the fitted line (with $R^{2}=0.9921$ ). FOV size is $9 \times 9 \mathrm{~cm}^{2}$. The Euclidean distance between neighboring samples is $1.7 \mathrm{~cm}$.
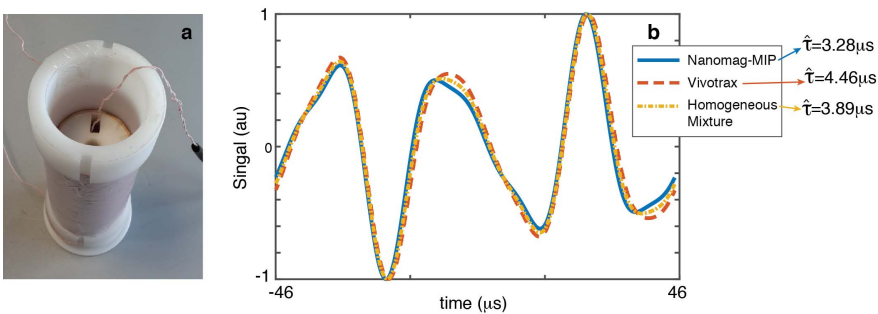

Fig. 8. Experimental demonstration in the MPS setup. (a) The picture of our in-house MPS setup, showing the drive and receive coils placed co-axially. (b) The signal acquired from Nanomag-MIP (blue) and VivoTrax (orange) SPIOs, and their homogeneous mixture (yellow). The Nanomag-MIP and VivoTrax SPIOs yielded $\hat{\tau}=3.28 \mu \mathrm{s}$ and $\hat{\tau}=4.46 \mu \mathrm{s}$, respectively. The homogeneous mixture gave $\hat{\tau}=3.89 \mu \mathrm{s}$, closely matching the expected value of $3.87 \mu$ s based on the average of the Nanomag-MIP and VivoTrax relaxation times. These experiments were conducted at $10.8 \mathrm{kHz}$ and $15 \mathrm{mT}$-peak drive field.

homogeneous mixture case. As given in Fig. 8b, the $\tau$ values for Nanomag-MIP and VivoTrax SPIOs were estimated as $3.28 \mu \mathrm{s}$ and $4.46 \mu \mathrm{s}$, respectively. Since the MPS signal levels of the SPIOs were equalized beforehand, the expected $\tau$ for the homogeneous mixture was equal to $3.87 \mu$ s, i.e., the average of the $\tau$ values for Nanomag-MIP and VivoTrax. The estimation revealed a relaxation time of $3.89 \mu \mathrm{s}$, closely matching the expected value (see Appendix for a detailed explanation of the homogeneous mixture case).

\section{Imaging Experiment Results}

Figure 9 displays the 1D imaging results of the proposed technique. In this experiment, a phantom with 3 tubes, each containing a different type of SPIO was imaged. To enable

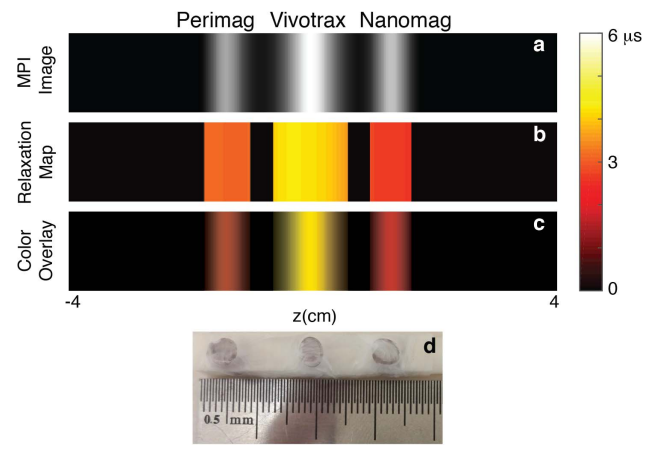

Fig. 9. Calibration-free multi-color MPI results in 1D. The imaging experiment was conducted at $9.7 \mathrm{kHz}$ and $15 \mathrm{mT}$-peak drive field, using three different nanoparticles. (a) The 1D reconstructed $x$-space MPI image, and (b) the corresponding multi-color relaxation map. (c) The color overlay of the MPI image and the multi-color relaxation map shows that the nanoparticles can be clearly distinguished based on their relaxation responses. (d) A photo of the imaged phantom with three tubes containing Perimag, VivoTrax, and Nanomag-MIP SPIOs from left to right, separated at $8 \mathrm{~mm}$ distances.

color display, the reconstructed 1D x-space MPI image and the $1 \mathrm{D}$ relaxation map were replicated and stacked vertically in a pseudo-2D image format. Figure 9a shows the reconstructed MPI image. Although the SPIOs were placed in identical tubes, VivoTrax covers a wider region $(7.3 \mathrm{~mm})$ in the MPI image, denoting a lower resolution capability. On the other hand, Perimag and Nanomag-MIP SPIOs display comparable widths ( $5 \mathrm{~mm}$ vs. $5.2 \mathrm{~mm}$ ).

While there are differences between the MPI responses of these SPIOs, it is not possible to distinguish the SPIO types based on the MPI image in Fig. 9a. Figure 9b shows the relaxation map reconstructed with the proposed algorithm. Here, different SPIO types can be clearly distinguished after color assignment. On average, Nanomag-MIP, Perimag, and VivoTrax SPIOs yielded $2.67 \mu \mathrm{s}, 3.02 \mu \mathrm{s}$, and $4.15 \mu \mathrm{s}$ relaxation time constants, respectively. Finally, Fig. 9c shows the color overlay image, containing the spatial and the quantitative information from both the MPI image and the relaxation map.

Figure 10 displays the 2D experimental demonstration of the proposed method. Here, Nanomag-MIP and VivoTrax SPIOs, and their homogeneous mixture were imaged with a $15 \mathrm{~mm}$ separation. Again, while the two SPIOs were placed in identical tubes, VivoTrax displays a significantly wider PSF in both $\mathrm{x}^{-}$and $\mathrm{z}$-directions in the MPI image in Fig. 10a. Accordingly, the homogeneous mixture shows less blurring than VivoTrax but larger blurring than Nanomag-MIP. In both the relaxation map and the color overlay image, NanomagMIP and VivoTrax SPIOs, and their homogeneous mixture can be distinguished clearly. The average time constants were measured as $2.82 \mu \mathrm{s}, 3.97 \mu \mathrm{s}$ and $3.57 \mu \mathrm{s}$, respectively. Here, due to the differences in the SPIO responses under a gradient field, the MPI signal ratio of VivoTrax to Nanomag-MIP was 1.6:1. Considering this signal ratio, the expected relaxation time constant of the homogeneous mixture was $3.53 \mu \mathrm{s}$, which closely matches the experimental result of $3.57 \mu \mathrm{s}$.

In Fig. 10b, at the peripheries of the SPIO distributions along the $\mathrm{x}$-direction, the estimated relaxation times deviate with respect to those in more central regions. While this is an 


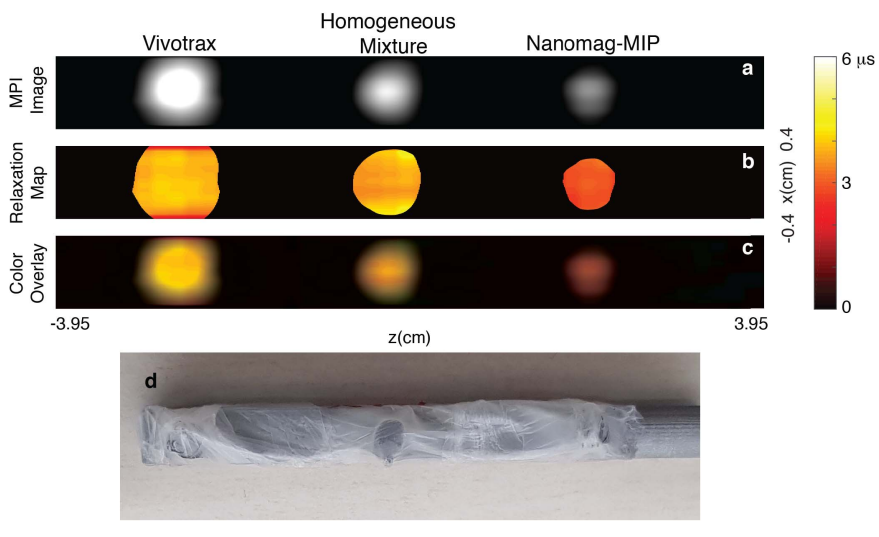

Fig. 10. Calibration-free multi-color MPI results in 2D. The imaging experiment was conducted at $9.7 \mathrm{kHz}$ and $15 \mathrm{mT}$-peak drive field, using Nanomag-MIP and VivoTrax SPIOs, and their homogeneous mixture. (a) The reconstructed $\mathrm{x}$-space MPI image and (b) the corresponding multi-color relaxation map. (c) The color overlay of the MPI image and the multi-color relaxation map shows that the two nanoparticles and their homogeneous mixture can be clearly distinguished in 2D, solely based on their relaxation responses. The mixture yields a $\tau$ value that closely matches the signal-weighted average of the $\tau$ values from NanomagMIP and VivoTrax. (d) A photo of the imaged phantom with three tubes containing VivoTrax, the homogeneous mixture, and Nanomag-MIP from left to right, separated at $15 \mathrm{~mm}$ distances.

undesired effect of low MPI signal, color overlay image naturally eliminates these low-pixel-intensity regions to provide a clean multi-color MPI image, as seen in Fig. 10c.

\section{Discussion}

Multi-color MPI is a very promising extension of MPI due to its potential use in cardiovascular interventions, temperature mapping, and viscosity mapping. There has been significant progress in multi-color MPI, both via SFR and $\mathrm{x}$ space reconstruction techniques. While these methods were shown to work exceptionally well, they either require extensive calibration for every SPIO types used [22] or multiple scans of the imaging FOV at different drive field amplitudes [35]. In this work, we have proposed and experimentally demonstrated a calibration-free approach for multi-color MPI, that works successfully with a single scan at a single drive field amplitude. Note that while relaxation times can be measured in a calibration-free fashion, for certain applications such as viscosity or temperature mapping, one must obtain prior fiducial measurements to create a dictionary of relaxation times corresponding to specific environmental conditions. These measurements could be performed on an MPS setup within seconds, or on an MPI scanner within minutes. Alternatively, these measurements could be obtained directly during imaging via placing the fiducials next to the object of interest and imaging with a slightly extended FOV.

\section{A. Effects of SPIO Types and Imaging Parameters}

Distinguishable SPIO behavior is a fundamental requirement for any multi-color MPI technique. SFR-based methods have the advantage of being able to distinguish nanoparticles with different responses, independent of whether that difference stems from the harmonic or the relaxation responses.
Still, Rahmer et al. [22], have stated that, SPIOs with similar size distributions or similar hysteresis behavior would be difficult to separate. In the case of the proposed method, SPIOs are required to have distinct relaxation time constants for a clear differentiation. For the experimental results shown in this work, all three nanoparticles involved were multicore SPIOs, with Perimag and Nanomag-MIP having similar chemical compositions and hydrodynamic diameters. Despite these similarities, the proposed multi-color MPI technique successfully distinguished these nanoparticles. To further improve the performance of this technique, two different approaches can be undertaken: SPIOs that are specifically tailored to have different relaxation behaviors would facilitate relaxation mapping. In addition, imaging parameters can be optimized to maximize the contrast between relaxation times. A previous work has compared the relaxation times of multi-core Resovist nanoparticles with single-domain UW33 nanoparticles, which had significantly different relaxation times. Interestingly, the ratio of the relaxation times remained almost constant (around 2-2.5) across a wide range of frequencies and drive field amplitudes [40]. These results suggest that, for such cases, the proposed method can be successfully applied at a variety of drive field parameters.

\section{B. Resolution of the Relaxation Map}

The pixel size in the relaxation map is equal to the distance between adjacent pFOV centers, which is a function of both the drive field amplitude and the overlap percentage. For the 2D imaging example in this work, this resolution is equal to approximately $1.9 \mathrm{~mm}$ along the $\mathrm{z}$-direction and $1 \mathrm{~mm}$ along the $\mathrm{x}$-direction. Note, however, that the resolution of our MPI scanner is approximately $4 \mathrm{~mm}$ along the $\mathrm{z}$-direction (for Nanomag-MIP nanoparticles). Hence, the true resolution of the relaxation map is still equal to the resolution of the MPI system, as that is the dominant factor under the given imaging conditions (see Fig. S4 in Supplementary Materials for a detailed resolution analysis).

For MPI systems that have higher gradient selection fields or when using MPI-optimized nanoparticles, the resolution of the relaxation map may be worse than that of the MPI system. One method to mitigate this problem would be to use high overlap percentages. While this solution may sound unpractical, it is actually necessitated by the human safety limits [8]-[12], [45]. Operating at the safety limits, a linearly-ramping focus field with $20 \mathrm{~T} / \mathrm{s}$ slew rate together with a $7 \mathrm{mT}$-peak drive field at $25 \mathrm{kHz}$ results in $97 \%$ overlap between adjacent pFOVs. With that said, it is not uncommon for functional imaging techniques to possess lower resolution than their anatomical counterparts. For example, functional Magnetic Resonance Imaging (MRI) or diffusion-weighted MRI both have significantly lower resolutions when compared to anatomical MRI.

\section{Fine Tuning of the Hardware Delays}

In this work, the $t_{\text {edge }}$ parameter achieves a fine tuning of the system delay (e.g., correction of sub-sample delays), as needed for accurate relaxation time estimation. A prior 
calibration of the global hardware delay can further increase the speed of the search algorithm via restricting the possible

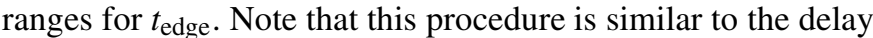
calibration procedure in MRI scanners. The delay between the applied gradient fields and data acquisition is calibrated globally for every MRI scanner, individually. Still, certain imaging procedures such as echo planar imaging (EPI) can be particularly sensitive to even small amounts of inaccuracies in this delay. Hence, a fine tuning of delays is performed every time an EPI image is acquired, using a reference scan data [46]. For the method proposed in this work, neither a global calibration nor a reference scan is necessitated, as the delay can be estimated simultaneously with the relaxation time constant.

\section{Towards True Quantitative MPI}

While quantitative imaging is a desirable feature in MPI, environmental factors such as viscosity and temperature, as well as the usage of different nanoparticle types may complicate quantitative imaging of iron concentration. SFRbased multi-color MPI approaches restore quantitativeness via individually calibrating the responses for different nanoparticles/conditions [22]. The technique proposed in this work can also be extended to restore quantitativeness for $\mathrm{x}$-space MPI images. For a fixed nanoparticle type, the effects of environmental factors can be reversed by deconvolving the signals with the estimated relaxation kernels to yield a quantitative MPI image. In the case of different nanoparticles, the multi-color relaxation map can be used to generate separate $\mathrm{x}$-space MPI images of each nanoparticle type, which can then be deconvolved by their respective measured PSFs and recombined to form a quantitative MPI image. For this latter approach, the PSFs could be measured either via a prior MPS measurement performed within seconds or by incorporating point source fiducials into the imaging volume, with a slight increase in scan time. Alternatively, a phantom made of point source fiducials could be imaged separately within a few minutes. Note that for SFR-based multi-color MPI approaches, such calibration scans last for hours for each nanoparticle type or environmental condition.

\section{E. Extensions to 2D/3D Excitation and FFL Scanners}

The Lissajous trajectory is a widely popular and efficient way of scanning the imaging volume due to its short imaging durations and comprehensive coverage [47], [48]. For the proposed method to work for $2 \mathrm{D} / 3 \mathrm{D}$ excitations such as the Lissajous trajectory, the FFP trajectory can be modified to produce data in opposing scan directions. Assuming that the non-adiabatic MPI signal model in [37] still applies, this modification could be achieved by scanning the same volume twice, with the excitation fields reversed in the second round. The signal could then be analyzed in local chunks to generate multi-color images.

The proposed method is not necessarily designed for FFP scanners and could potentially be extended to FFL scanners, after necessary modifications. Previous work has demonstrated that the non-adiabatic MPI signal model also applies in the case of 1D excitation in an FFL system [49]. Hence, we expect the underlying time constant estimation method to work as it is. However, akin to the inhomogeneous mixture case, signal contributions from different regions along the FFL may result in incorrect relaxation time constant estimations. Hence, similar to Phase 4 of the proposed algorithm, a recovery scheme must be developed to handle those special cases. A subsequent reconstruction algorithm based on filtered backprojection can be used to reconstruct 3D multi-color images. Extending the proposed method to trajectories with multidimensional drive fields or FFL scanners remain as important future works.

\section{CONCLUSION}

In this work, we have proposed a calibration-free multicolor MPI technique and demonstrated the results via both MPS and imaging experiments. The proposed technique relies on distinguishing nanoparticles based on their relaxation responses, where the relaxation times are directly estimated from the MPI signal via restoring the underlying mirror symmetry. A step-by-step algorithm is proposed to generate an accurate relaxation map (or multi-color MPI image) from the time constant estimations for each pFOV. The imaging results show that different nanoparticle responses can be successfully distinguished, without any calibration or prior information regarding their responses. The proposed calibrationfree multi-color MPI technique is a promising method for future functional imaging applications of MPI, such as catheter tracking, viscosity mapping, temperature mapping, and stem cell tracking.

\section{APPENDIX Relaxation Time CONStant of A HOMOGENEOUS MIXTURE}

As stated in Section III-C, when two different SPIOs are mixed homogeneously, $\hat{\tau}$ is the weighted average of the relaxation time constants of the two SPIOs. Here, we assume that the mixture is completely homogeneous and the two SPIOs have identical adiabatic signals, $s_{\text {half }}(t)$ (e.g., bound/unbound SPIOs with identical nanoparticle core sizes). Therefore, Eqs. 5 and 6 can be modified as follows:

$$
\begin{aligned}
& s_{\text {pos }}(t)=c_{1} s_{\text {half }}(t) * r_{1}(t)+c_{2} s_{\text {half }}(t) * r_{2}(t) \\
& s_{\text {neg }}(t)=c_{1}\left(-s_{\text {half }}(-t) * r_{1}(t)\right)+c_{2}\left(-s_{\text {half }}(-t) * r_{2}(t)\right)
\end{aligned}
$$

Here, $c_{1}$ and $c_{2}$ are the concentrations of the two different SPIOs, and $r_{1}(t)$ and $r_{2}(t)$ are the corresponding relaxation kernels with $\tau_{1} \neq \tau_{2}$. Fourier domain representation of Eqs. 13 and 14 can be written as follows:

$$
\begin{aligned}
R_{j}(f) & =\frac{1}{1+i 2 \pi f \tau_{j}}, \quad j=1,2 \\
S_{\mathrm{pos}}(f) & =S_{\text {half }}(f) \cdot\left(c_{1} R_{1}(f)+c_{2} R_{2}(f)\right) \\
S_{\text {neg }}(f) & =-S_{\text {half }}^{*}(f) \cdot\left(c_{1} R_{1}(f)+c_{2} R_{2}(f)\right)
\end{aligned}
$$

Here, there are three unknowns, $S_{\text {half }}, \tau_{1}$, and $\tau_{2}$, and two equations, Eq. 16 and 17. Hence, the equation set above is underdetermined. Nevertheless, if the original estimation 
method given in Eq. 10 is applied, the following expression can be obtained using Eqs. 16-17.:

$$
\begin{aligned}
\tau(f) & =\frac{S_{\text {pos }}^{*}(f)+S_{\text {neg }}(f)}{i 2 \pi f\left(S_{\text {pos }}^{*}(f)-S_{\text {neg }}(f)\right)} \\
& =\frac{2 c_{1} \tau_{1}\left(1+4 \pi^{2} f^{2} \tau_{2}^{2}\right)+2 c_{2} \tau_{2}\left(1+4 \pi^{2} f^{2} \tau_{1}^{2}\right)}{2 c_{1}\left(1+4 \pi^{2} f^{2} \tau_{2}^{2}\right)+2 c_{2}\left(1+4 \pi^{2} f^{2} \tau_{1}^{2}\right)}
\end{aligned}
$$

Equation 18 is not strictly linear with respect to concentrations of the SPIOs in the mixture. However, for small frequencies (e.g., for $f<4 f_{0}$ where $f_{0}$ is the drive field frequency), the terms $\left(1+4 \pi^{2} f^{2} \tau_{\mathrm{i}}^{2}\right)$ can be approximated as 1 . In that case, Eq. 18 simplifies to the following form:

$$
\tau(f) \approx \frac{c_{1} \tau_{1}+c_{2} \tau_{2}}{c_{1}+c_{2}}
$$

where $\tau(f)$ is equal to the weighted average of $\tau_{1}$ and $\tau_{2}$. Simulation results presented in Fig. $7 \mathrm{~d}$ confirm the validity of Eq. 19, i.e., the estimation behaves linearly with respect to relaxation time constants and the relative concentrations in the mixture.

Note that, if the adiabatic responses of the two SPIOs are different (e.g., SPIOs with different core sizes), the estimated $\tau(f)$ will be an average of $\tau_{1}$ and $\tau_{2}$, weighted by the overall signal levels from the two SPIOs. In addition, an SPIO sample may exhibit a spread of $\tau$ values, e.g., due to polydispersity in nanoparticle size. For that case, the estimated relaxation time constant will be an average over the spread of $\tau$ values, weighted by their relative signal contributions.

\section{REFERENCES}

[1] B. Gleich and J. Weizenecker, "Tomographic imaging using the nonlinear response of magnetic particles," Nature, vol. 435, pp. 1214-1217, Jun. 2005

[2] J. Weizenecker, J. Borgert, and B. Gleich, "A simulation study on the resolution and sensitivity of magnetic particle imaging," Phys. Med. Biol., vol. 52, no. 21, pp. 6363-6374, 2007.

[3] P. W. Goodwill et al., "X-space MPI: Magnetic nanoparticles for safe medical imaging," Adv. Mater., vol. 24, no. 28, pp. 3870-3877, Jul. 2012.

[4] E. U. Saritas et al., "Magnetic particle imaging (MPI) for NMR and MRI researchers," J. Magn. Reson., vol. 229, pp. 116-126, Apr. 2013.

[5] L. M. Bauer, S. F. Situ, M. A. Griswold, and A. C. S. Samia, "Magnetic particle imaging tracers: State-of-the-art and future directions," J. Phys. Chem. Lett, vol. 6, no. 13, pp. 2509-2517, 2015.

[6] K. Lu, P. W. Goodwill, E. U. Saritas, B. Zheng, and S. M. Conolly, "Linearity and shift invariance for quantitative magnetic particle imaging," IEEE Trans. Med. Imag., vol. 32, no. 9, pp. 1565-1575, Sep. 2013.

[7] B. Zheng et al., "Quantitative magnetic particle imaging monitors the transplantation, biodistribution, and clearance of stem cells in vivo," Theranostics, vol. 6, no. 3, pp. 291-301, 2016.

[8] I. Schmale et al., "Human PNS and SAR study in the frequency range from 24 to $162 \mathrm{kHz}$," in Proc. 3rd Int. Workshop Magn. Particle Imag., Berkeley, CA, USA, Mar. 2013, p. 1.

[9] E. U. Saritas, P. W. Goodwill, G. Z. Zhang, and S. M. Conolly, "Magnetostimulation limits in magnetic particle imaging," IEEE Trans. Med. Imag., vol. 32, no. 9, pp. 1600-1610, Sep. 2013

[10] I. Schmale, B. Gleich, J. Rahmer, C. Bontus, J. Schmidt, and J. Borgert, "MPI safety in the view of MRI safety standards," IEEE Trans. Magn, vol. 51, no. 2, Feb. 2015, Art. no. 6502604.

[11] E. U. Saritas, P. W. Goodwill, and S. M. Conolly, "Effects of pulse duration on magnetostimulation thresholds," Med. Phys., vol. 42, no. 6, pp. 3005-3012, 2015

[12] O. B. Demirel and E. U. Saritas, "Effects of duty cycle on magnetostimulation thresholds in MPI," Int. J. Magn. Particle Imag., vol. 3, no. 1, p. $1703010,2017$.
[13] P. W. Goodwill, "Projection X-space magnetic particle imaging," IEEE Trans. Med. Imag., vol. 31, no. 5, pp. 1076-1085, May 2012.

[14] P. Goodwill et al., Third Generation X-Space MPI Mouse and Rat Scanner. Berlin, Germany: Springer, 2012, pp. 261-265.

[15] P. W. Goodwill et al., "A 7 T/M 3D X-space MPI mouse and rat scanner," in Proc. 3rd Int. Workshop Magn. Particle Imag., Berkeley, CA, USA, Mar. 2013, p. 1

[16] J. Rahmer et al., "Signal encoding in magnetic particle imaging: Properties of the system function," BMC Med. Imag., vol. 9, p. 4, Dec. 2009.

[17] T. Knopp et al., "Model-based reconstruction for magnetic particle imaging," IEEE Trans. Med. Imag., vol. 29, no. 1, pp. 12-18, May 2010.

[18] T. Knopp et al., "Weighted iterative reconstruction for magnetic particle imaging," Phys. Med. Biol., vol. 55, no. 6, pp. 1577-1589, 2010.

[19] J. Rahmer, "Analysis of a 3-D system function measured for magnetic particle imaging," IEEE Trans. Med. Imag., vol. 31, no. 6, pp. 1289-1299, Jan. 2012.

[20] P. W. Goodwill and S. M. Conolly, "The X-space formulation of the magnetic particle imaging process: 1-D signal, resolution, bandwidth, SNR, SAR, and magnetostimulation," IEEE Trans. Med. Imag., vol. 29, no. 11, pp. 1851-1859, Nov. 2010.

[21] P. W. Goodwill and S. M. Conolly, "Multidimensional X-space magnetic particle imaging," IEEE Trans. Med. Imag., vol. 30, no. 9, pp. 1581-1590, Sep. 2011.

[22] J. Rahmer, A. Halkola, B. Gleich, I. Schmale, and J. Borgert, "First experimental evidence of the feasibility of multi-color magnetic particle imaging," Phys. Med. Biol., vol. 60, no. 5, pp. 1775-1791, Feb. 2015.

[23] J. Haegele et al., "Magnetic particle imaging: A resovist based marking technology for guide wires and catheters for vascular interventions," IEEE Trans. Med. Imag., vol. 35, no. 10, pp. 2312-2318, Oct. 2016.

[24] N. Nothnagel, J. Rahmer, B. Gleich, A. Halkola, T. M. Buzug, and J. Borgert, "Steering of magnetic devices with a magnetic particle imaging system," IEEE Trans. Biomed. Eng., vol. 63, no. 11, pp. 2286-2293, Nov. 2016.

[25] J. Rahmer, D. Wirtz, C. Bontus, J. Borgert, and B. Gleich, "Interactive magnetic catheter steering with 3-D real-time feedback using multi-color magnetic particle imaging," IEEE Trans. Med. Imag., vol. 36, no. 7, pp. 1449-1456, Jul. 2017.

[26] C. Stehning, B. Gleich, and J. Rahmer, "Simultaneous magnetic particle imaging (MPI) and temperature mapping using multi-color MPI," Int. J. Magn. Particle Imag., vol. 2, no. 2, p. 1612001, 2016

[27] A. M. Rauwerdink and J. B. Weaver, "Measurement of molecular binding using the Brownian motion of magnetic nanoparticle probes," Appl. Phys. Lett, vol. 96, no. 3, p. 033702, 2010.

[28] S. Bhaskar et al., "Multifunctional Nanocarriers for diagnostics, drug delivery and targeted treatment across blood-brain barrier: Perspectives on tracking and neuroimaging," Part Fibre Toxicol, vol. 7, p. 3, Mar. 2010.

[29] A. M. Rauwerdink and J. B. Weaver, "Viscous effects on nanoparticle magnetization harmonics," J. Magn. Magn. Mater, vol. 322, no. 6, pp. 609-613, 2009.

[30] J. B. Weaver, A. M. Rauwerdink, and E. W. Hansen, "Magnetic nanoparticle temperature estimation," Med. Phys., vol. 36, no. 5, pp. 1822-1829, 2009.

[31] T. Wawrzik et al., "Effect of brownian relaxation in frequency-dependent magnetic particle spectroscopy measurements," in Proc. 3rd Int. Workshop Magn. Particle Imag., Berkeley, CA, USA, Mar. 2013, p. 1.

[32] M. Hofmann, J. Dieckhoff, H. Ittrich, and T. Knopp, "Experimental distinction of different viscosities using multispectral magnetic particle imaging," in Proc. 6th Int. Workshop Magn. Particle Imag., Luebeck, Germany, 2016, p. 9.

[33] M. Utkur, Y. Muslu, and E. U. Saritas, "Relaxation-based viscosity mapping for magnetic particle imaging," Phys. Med. Biol, vol. 62, no. 9, pp. 3422-3439, 2017.

[34] T. Viereck, C. Kuhlmann, S. Draack, M. Schilling, and F. Ludwig, "Dual-frequency magnetic particle imaging of the Brownian particle contribution," J. Magn. Magn. Mater, vol. 427, pp. 156-161, Apr. 2017.

[35] D. Hensley, P. Goodwill, L. Croft, and S. Conolly, "Preliminary experimental x-space color MPI," in Proc. 5th Int. Workshop Magn. Particle Imag., Istanbul, Turkey, Mar. 2015, p. 1.

[36] Y. Muslu, M. Utkur, O. B. Demirel, and E. U. Saritas, "Calibration-free color MPI," in Proc. 6th Int. Workshop Magn. Particle Imag., Luebeck, Germany, 2016, p. 10.

[37] L. R. Croft, P. W. Goodwill, and S. M. Conolly, "Relaxation in X-space magnetic particle imaging," IEEE Trans. Med. Imag., vol. 31, no. 12, pp. 2335-2342, Dec. 2012 
[38] P. Debye, "Polar molecules. by p. debye, ph.d., pp. 172. New York: Chemical Catalog co., inc., 1929," J. Soc. Chem. Ind., vol. 48, no. 43, pp. 1036-1037, 1929.

[39] M. I. Shliomis, "Magnetic fluids," Phys. Uspekhi, vol. 17, no. 2, pp. 153-169, 1974.

[40] L. R. Croft et al., "Low drive field amplitude for improved image resolution in magnetic particle imaging," Med. Phys, vol. 43, no. 1, pp. 424-435, 2015.

[41] G. Onuker and E. U. Saritas, "Simultaneous relaxation estimation and image reconstruction in MPI," in Proc. 5th Int. Workshop Magn. Particle Imag., Istanbul, Turkey, Mar. 2015, p. 1.

[42] J. J. Konkle, P. W. Goodwill, D. W. Hensley, R. D. Orendorff, M. Lustig, and S. M. Conolly, "A convex formulation for magnetic particle imaging X-space reconstruction," PLOS ONE, vol. 10, no. 10, p. e0140137-15, 2015.

[43] J. MacQueen, "Some methods for classification and analysis of multivariate observations," in Proc. 5th Berkeley Symp. Math. Statist. Probab., vol. 1. 1967, pp. 281-297.

[44] R. M. Ferguson et al., "Magnetic particle imaging with tailored iron oxide nanoparticle tracers," IEEE Trans. Med. Imag., vol. 34, no. 5, pp. 1077-1084, May 2015.
[45] J. J. Konkle, P. W. Goodwill, E. U. Saritas, B. Zheng, K. Lu, and S. M. Conolly, "Twenty-fold acceleration of 3D projection reconstruction MPI," Biomed. Technik/Biomed. Eng., vol. 58, no. 6, pp. 565-576, 2013.

[46] H. Bruder, H. Fischer, H.-E. Reinfelder, and F. Schmitt, "Image reconstruction for echo planar imaging with nonequidistant $k$ space sampling," Magn. Reson. Med., vol. 23, no. 2, pp. 311-323, 1992.

[47] C. Kaethner, W. Erb, M. Ahlborg, P. Szwargulski, T. Knopp, and T. M. Buzug, "Non-equispaced system matrix acquisition for magnetic particle imaging based on lissajous node points," IEEE Trans. Med. Imag., vol. 35, no. 11, pp. 2476-2485, Nov. 2016.

[48] T. Knopp, S. M. Conolly, and T. M. Buzug, "Recent progress in magnetic particle imaging: From hardware to preclinical applications," Phys. Med. Biol., vol. 62, no. 9, p. E4, 2017.

[49] K. Bente, M. Weber, M. Graeser, T. F. Sattel, M. Erbe, and T. M. Buzug, "Electronic field free line rotation and relaxation deconvolution in magnetic particle imaging," IEEE Trans. Med. Imag., vol. 34, no. 2, pp. 644-651, Feb. 2015. 